\title{
Development of Magnetic Field Sensor and Motor Fault Monitoring Application
}

\author{
Ziyuan Tong1,2, Zhaoyang Dong1*, Minming Tong2, Bo Wang2, Li Meng2 \\ ${ }^{1}$ School of Electrical and Information Engineering, University of Sydney, NSW, Australia \\ ${ }^{2}$ Engineering Institute of Information and Electricity, China University of Mining Technology, Xuzhou, China \\ Email: ${ }^{*}$ zton3078@uni.sydney.edu.au
}

Received March 2014

Copyright (C) 2014 by authors and Scientific Research Publishing Inc.

This work is licensed under the Creative Commons Attribution International License (CC BY). http://creativecommons.org/licenses/by/4.0/

(c) (i) Open Access

\begin{abstract}
For the purpose of motor fault real-time monitoring, this research developed a nano-silicon nitride film based magnetic field (MF) sensor, and applied this sensor in MF detection of two common faults. Through experiment, it turned out that arc discharge and slot discharge occur in motor fault produce MF with certain laws. This result proved the feasibility of the sensor and sensing method in MF analysis, and revealed possibility of a new method in fault detection.
\end{abstract}

Keywords

Magnetic Field Sensor, Motor Fault, Slot Discharge, Arc Discharge, Real-Time Monitoring

\section{Introduction}

The motor, in an event of a failure, may produce a spark discharge. This spark can be a direct source of fire, and it can also damage power instruments because of the heat generated in discharge. The electric field and magnetic field strengths will have some certain levels of changes. By sensing and analyzing the feature of changing intensity, we can monitor and diagnose the faults in an operating motor. In the detection procedure, we need magnetic field sensing elements, which can accurately obtain the MF intensity on a specific point. In this paper, we describe the procedure of sensor development, and use the sensor manufactured in magnetic field analyzing.

\section{Development of MF Sensor}

The structure of MF sensor is shown in Figure 1. The working principle is based on electro-magnetic coupling relationship. In this sensor, magnetic-sensing film is used to generate diffusion potential in magnetic field. This potential leads to the change in current carrier in channel $\mathrm{N}$, which results in current signal variation between

*Corresponding author. 


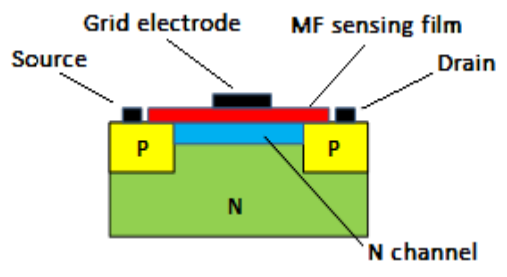

Figure 1. MF sensor structure.

drain electrode and source electrode. This process helps to achieve the MF detection.

Fabrication procedure of MF sensor includes substrate preparation, oxidation, photolithography erosion, film deposition, fluorescent purification, electrodes sputtering, and slicing, sintering, bonding, sealing [1].

Firstly, we deposited $20 \mathrm{~nm}$ of nano-silicon nitride film on epitaxial wafer of substrate through Pulsed Laser Deposition (PLD). In the deposition process, we kept the substrate temperature at $800^{\circ} \mathrm{C}$. After deposition, we applied photo etching to the deposition layer. Then we uniformly dispersed the positive photoresist with spincoating method on in the film. After that we exposed the film with the photolithography and remove the photoresist. This process is followed by corrosion. Then we examined the regularity of lithography line under microscope [2].

After the photo etching is finished, we need to do plasma etching. The main purpose is to remove the dirt on the film surface. We uniformly disperse the negative photoresist with spin-coating method on in the film. Then we exposed the film with the second photolithography. We plated 2 - 3 um of gold on the film with thermal evaporation method.

After the gold film has been evaporated, we put the film with negative photoresist and gold film into acetone solvent, heated the solvent for 4 - 5 minutes, and observed the gold electrode. When the electrode was fully visible, we did plasma etching with oxygen plasma to completely remove the photoresist. Then the grating type fieldtron has been successfully fabricated on the N-Si substrate. Finally we use ultrasonic bonding to package the device, welding the leads to pins. After we finished all the procedures above, the MF sensor was fabricated.

\section{MF Sensor Applications in Motor Fault Discharge Detection}

\subsection{Slot Discharge Detection}

Due to the core vibration in motor working produces, it is possible that fault occurs on the fixed parts of stators, such as antihalation layer damaged and slot carve loosened. These may lead to insufficient thermal expansion of insulation at different temperatures, and slot wall and slot part may have poor contact with each other accordingly. As a result, the slot bottom or slot wall will not have good contact with stator bar. When the electric field in gap is large enough to cause a breakdown, there will be a slot discharge [3].

Figure 2 shows the physical model of slot discharge. In this model, copper plate is connected to ground and copper core is connected to high voltage, and there is an air gap created by low resistance paint layer between stator bar and the insulation. With this structure, we can simulate the discharge caused by poor contact condition between bar and slot wall.

We regulated the transformer, and observe the phenomenon on different discharge levels. When the power supply voltage is about $45 \mathrm{kV}$, the output voltage signal reaches $4 \mathrm{~V}$. The measured amplitude-frequency characteristic is shown in Figure 3.

We can see from the signal waveform that, the initial amplitude is large, but it damps quickly. Then the amplitude fluctuates in a small range It is shown in amplitude-frequency wave that the signal is mainly distributed in the range of 0 - $250 \mathrm{kHz}$.

\subsection{Arc Discharge Detection}

When the motor stator winding is working under mechanical, electricity, heat and other forces, the plied wire of stator winding may rupture, which will cause arc discharge. Because of the zero crossing character of power frequency current, this form of discharge will repeatedly extinguish and rekindle with great energy conversion. Heat generated in conversion process will accelerate insulation ageing and have significant harm to motor protection system [4]. Figure 4 shows the physical model of arc discharge. 
We regulated the transformer until the secondary side output voltage reaches about $40 \mathrm{kV}$. The discharge MF has been large enough, and the induced voltage signal accordingly has been strong. The measured amplitude-frequency characteristic is shown in Figure 5.

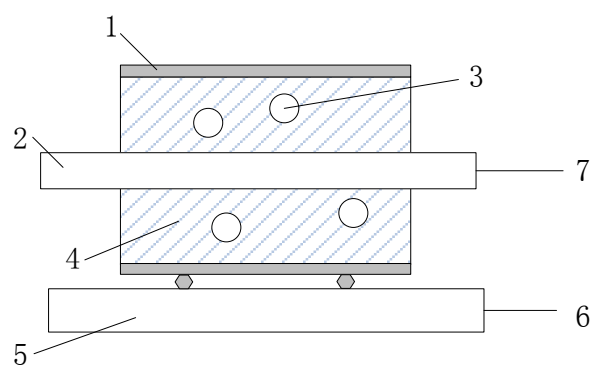

Figure 2. Motor slot discharge model. 1-antihalation layer; 2-winding bar; 3-air gap; 4-insulation; 5-stator core; 6-ground terminal; 7high voltage terminal.

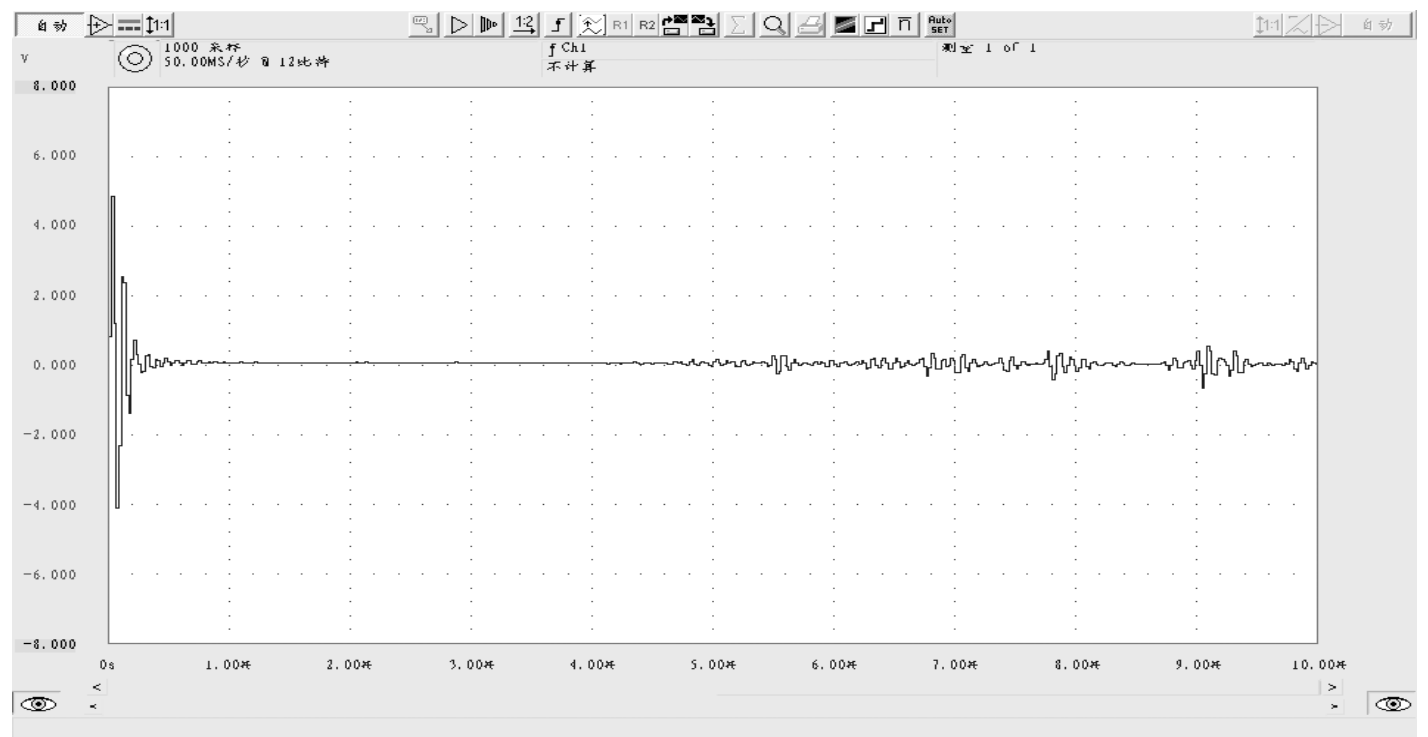

Figure 3. MF signal of motor slot discharge in frequency domain.

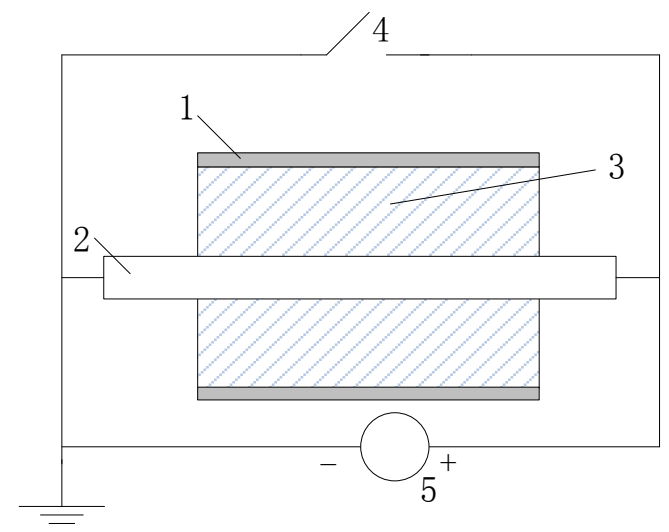

Figure 4. Motor arc discharge model. 1-antihalation layer; 2-winding bar; 3-insulation; 4-electronic switch; 5-current source. 


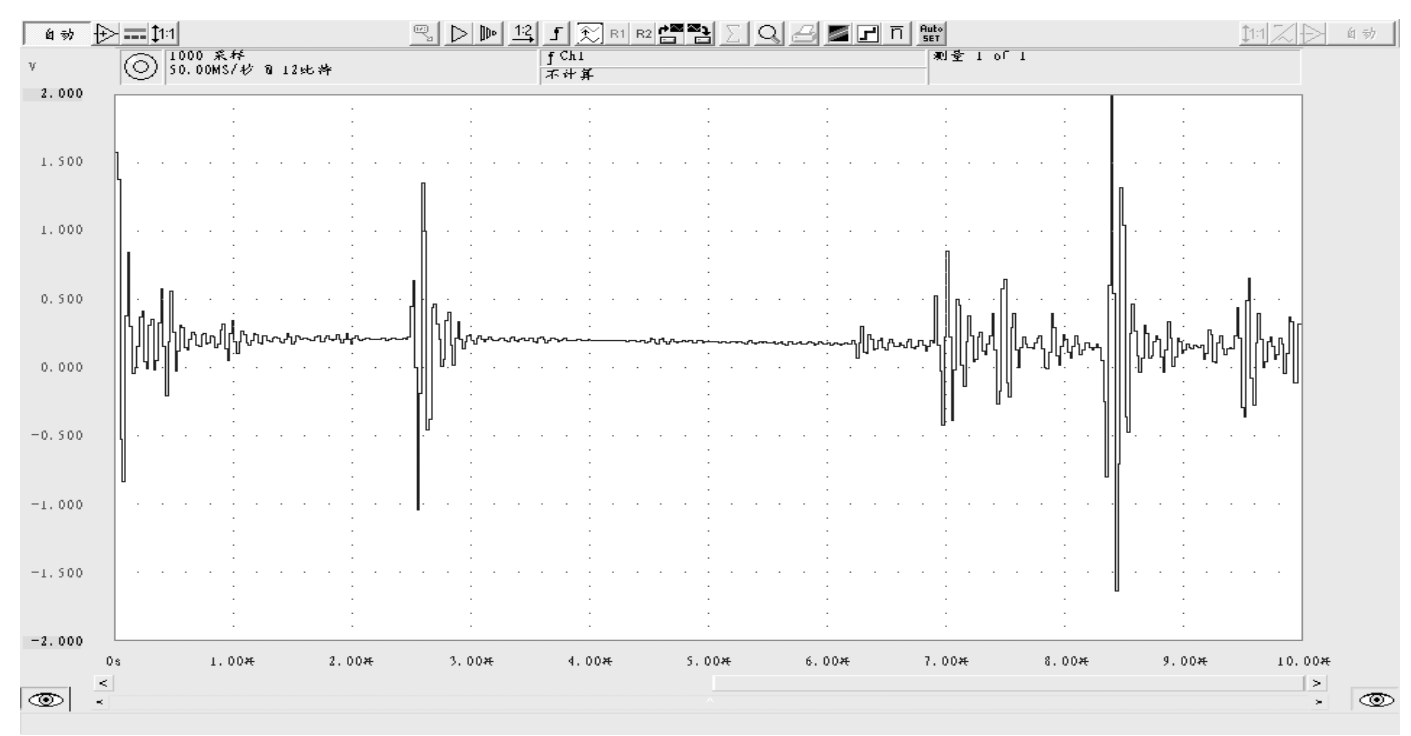

Figure 5. MF signal of motor arc discharge in frequency domain.

We can see from the signal waveform that, the amplitude increases in initial period and reaches the first peak of $1 \mathrm{~V}$. After short durations, the second and the third wave clusters appear. The third wave cluster has the largest amplitude and duration. The characters of arc discharge MF are much different from those of slot discharge signal. Arc discharge MF lasts longer with much noise. It is shown in amplitude-frequency wave that the signal is mainly distributed in the range of $0-750 \mathrm{kHz}$, so arc discharge signal has a wider band compared with slot discharge signal.

\section{Conclusions}

In this paper, a MF sensor has been developed for motor fault discharge detection. Through applying the sensor in collecting and analyzing MF signal of two discharge modes, we proved this sensor feasible in MF detection.

From the analyzing results, we can see that signal of motor slot discharge is strong but the duration is short, while arc discharge signal has a high peak and the signal lasts longer. The initiation signal strength of arc discharge is weak, and it can increase sharply in a short period. Slot discharge signal continues for a long time with much noise.

Two discharge modes has obvious difference in amplitudes, based on which we can easily distinguish the fault types.

\section{References}

[1] Macintyre, S.A. (1991) Magnetic Field Sensor Design, Sensor Review, 11, 7-11

[2] Zhou, Z.J., Cheng, D.F., Wang, J., Zhang, Z.Y. and Liu, X. (2009) Development of Magneto-Dependent Sensor in the Optical Pumped Magnetometer. Chinese Journal of Sensors and Actuators, 22, 1284-1288

[3] Wilson, A. (1990) High and Low Intensity Slot Discharge. Conference Record of IEEE International Symposium on Electrical Insulation, 363-366

[4] Hanazawa, T., Almazroui, A. and Egashira, T. (2003) The Distribution of Commutation Sparks in Universal Motors, Sangyo Oyo Bumonshi, 123, 1546. 